\title{
Not so neglected diseases
}

\author{
Roy D. Sleator \\ Alimentary Pharmabiotic Centre, University College Cork, College Road, Cork, Ireland.
}

J Infect Developing Countries 2007; 1(3):350-351.

Received 8 November 2007 - Accepted 2 December 2007.

Copyright $@ 2007$ Sleator. This is an open access article distributed under the Creative Commons Attribution License, which permits unrestricted use, distribution, and reproduction in any medium, provided the original work is properly cited.

Nature in its recent Outlook on Neglected diseases (Outlook: Neglected diseases 449, 157$182 ; 2007)$ focused new light on a subject which has itself been long neglected by the scientific community and in the scientific literature; that is, the Developing World's struggle with endemic infections (such as malaria) and epidemics (such as HIV (AIDS)) and the role of the Developed World in this struggle.

The benefits of the current "Push-Pull" approach as outlined in the Nature outlook piece (i.e., the provision of subsidies and guaranteed markets) to encourage the first world to break free of the traditional academic culture which rewards publications and wealth creation over contributions to the practical social good, thereby helping to bridge the "translational gap" between low- and high-income countries, are obvious and well presented [1].

However, an additive, if not alternative, approach to combating "neglected diseases", which has received considerably less attention, is the need to encourage researchers from the developing world to join the fray themselves. After all, tropical diseases are neither forgotten nor neglected by this cohort for whom the incentives are life-and-death as opposed to scientific recognition or wealth generation.

With this in mind, the most effective approaches to helping developing world researchers help themselves are, in my opinion, knowledge transfer through appropriate education and outreach programs and improved access to the international scientific literature.

To this end The National Institutes of Health (NIH) Office of Technology Transfer (OTT) has made significant advances in interdisciplinary training and mentoring of scientists from less well developed countries, in an initiative designed to foster innovation and encourage sustainable research efforts in developing countries. This approach, based on a novel mentoring system, allows scientists from developing countries to gain first-hand practical experience of a variety of key areas including technology transfer, licensing and commercialization of technologies [2].

In addition to improving research strength and capacity through novel mentoring programs, there is a growing recognition of the need to facilitate increased access to the international scientific literature [3]. This is a two-way street and involves not only improved access by developing world scientists to journals in order to obtain information, but also to make their own independent contributions to the body of scientific knowledge. This approach includes the introduction of online open access journals, such as our very own Journal of Infection in Developing Countries, which actively encourage submissions from developing world authors and engage supportive panels of editors to provide mentorship in improving articles with obvious scientific merit. It is hoped that this tactic will ultimately help to counter the current disequilibrium that exists in collaborative research between low- and high-income countries, where the role of the developing world researcher is often "operational" (involving routine data collection) as opposed to "intellectual" (study design and authorship) [4]. This approach will ultimately ensure elevation of scientists from developing countries within the international scientific community, improving opportunities for further research funding and collaboration. 
In conclusion, while significant advances have and continue to be made by scientists in the developed world in the fight against "neglected diseases", future battles might ultimately be fought and won by better informed researchers from the developing world itself.

\section{Acknowledgements}

Dr Roy D. Sleator is a Health Research Board (HRB) Principal Investigator.

\section{References}

1. O'Connell D (2007) Nature: Outlook Neglected diseases. 449: 157.

2. Salicrup LA (2007) NIH forges R\&D partnerships in developing countries. Nat Biotech. 25: 976-977.

3. Mason PR (2007) The need for a journal of infection in developing countries. J Infect Develop Countries. 1: 3-6.

4. Mony PK, Kurpad A, Vaz M. (2005) Capacity building in collaborative research is essential. BMJ 331: 843-844.

Corresponding Author: Roy Sleator, Alimentary Pharmabiotic Centre, University College Cork, College Road, Cork, Ireland. Phone 0035321 4901366, Fax 00 35321 4903101, e-mail: r.sleator@ucc.ie.

Conflict of interest: No conflict of interest is declared. 